\title{
Pseudoxanthoma Elasticum: An Interesting Model to Evaluate Chronic Kidney Disease-Like Vascular Damage without Renal Disease
}

\author{
Luis D’Marco $^{a}$ Marcos Lima-Martínez $^{\mathrm{b}} \quad$ Cristina Karohl $^{\mathrm{c}}$ Maricarmen Chacín $^{\mathrm{d}}$ \\ Valmore Bermúdez ${ }^{\mathrm{d}}$
}

${ }^{a}$ Nephrology Department, Hospital Clinico Universitario, INCLIVA, Valencia, Spain;

${ }^{b}$ Department of Physiological Sciences, Universidad de Oriente, Bolívar, Venezuela;

'Universidade Federal do Rio Grande do Sul, Faculdade de Medicina, Porto Alegre, Brazil;

dUniversidad Simón Bolívar, Facultad de Ciencias de la Salud, Barranquilla, Colombia

\section{Keywords}

Pseudoxanthoma elasticum - Chronic kidney disease .

Vascular calcification · Hydroxyapatite

\begin{abstract}
Background: Pseudoxanthoma elasticum (PXE; OMIM 264800) is an inherited multisystem disorder associated with accumulation of mineralized and fragmented elastic fibers in the skin, vascular walls, and brush membrane in the eye. Carriers exhibit characteristic lesions in the cardiovascular system, and peripheral and coronary arterial disease as well as mitral valvulopathy often present as a cardiovascular feature of this disease. PXE and chronic kidney disease (CKD) share some common patterns in the vascular damage and in therapeutic approaches as well. Summary: To date, treating PXE has focused more on careful follow-up examinations with retinal specialists and cardiologist, avoiding long-term anticoagulation. Like CKD, maintaining a low-calcium diet, increasing dietary magnesium, and administering phosphate binders such as aluminum hydroxide or sevelamer may yield a modest benefit. Recently, 4-phenylbutyrate acid (4-PBA) has demonstrated a maturation of ABCC6 mutant effects into the plasma membrane. Moreover, in a humanized mouse model of PXE, 4-PBA administration restored the
\end{abstract}

physiological function of ABCC6 mutants, resulting in enhanced calcification inhibition and thus a promising strategy for allele-specific therapy of ABCC6-associated calcification disorders. Key Message: Vascular compromise in PXE patients share some components similar to CKD.

C 2020 The Author(s)

Published by S. Karger AG, Basel

\section{Introduction}

Vascular calcification (VC) is an independent risk factor for cardiovascular disease. There are various degrees of vascular damage among patients with chronic kidney disease (CKD). Both traditional risk factors for atherosclerosis and factors more closely associated with progressive loss of kidney function contribute to increase the incidence of cardiovascular complications seen in these patients. Thus, 2 primary forms of arterial pathology develop in patients with CKD: atherosclerosis, with the accumulation of inflammatory cells, lipids, fibrous tissue, and calcium in the subintimal space, and arteriosclerosis [1]. The latter is characterized by a collection of hydroxyapatite deposits and amorphous calcium crystals in the vessel's tunica media and is believed to be more closely

\begin{tabular}{ll}
\hline KARGER & ( 2020 The Author(s) Karger \\
& Published by S. Karger AG, Basel Open caccess \\
karger@karger.com & This article is licensed under the Creative Commons Attribution- \\
NonCommercial-NoDerivatives 4.0 International License (CC BY- \\
NC-ND) (http://www.karger.com/Services/OpenAccessLicense). \\
Usage and distribution for commercial purposes as well as any dis- \\
tribution of modified material requires written permission.
\end{tabular}

Luis D'Marco, $\mathrm{MD}, \mathrm{PhD}$

Nephrology Department, Hospital Clinico Universitario, INCLIVA

Av. de Blasco Ibáñez, 17

ES-46010 Valencia (Spain)

E-Mail luisgerardodg@ hotmail.com 
associated with alterations in mineral metabolism than with traditional atherosclerosis risk factors [2]. The result is the development of what appears to be premature arterial aging with loss of elastic properties, increases stiffness, and increased overall fragility of the arterial system.

Pseudoxanthoma elasticum (PXE; OMIM 264800) is a rare genetic disorder caused by mutations in the ABCC6 gene which may function as a transporter and is mainly expressed in the kidneys and liver and characterized by elastorrhexia - a progressive calcification and fragmentation of elastic fibers in the skin (yellowish papules or plaques with "plucked chicken appearance"), retina (angioid streaks, peau d'orange, and choroidal neovascularization), and vessels (mainly by arterial calcification). In this sense, PXE is a considerable health threat due to severe visual impairment and blindness, peripheral arterial disease, ischemic stroke, mitral valve prolapse, and coronary artery disease resulting in angina pectoris and subsequent myocardial infarction $[3,4]$.

PXE has been found in people all over the world irrespective of their ethnic background, but a higher prevalence has been detected in some populations like "Afrikaners" from South Africa and some isolated families in the Netherlands $[5,6]$. Although PXE is a disease with high phenotypic variability and more than 300 mutations documented, the 4 most frequent mutations and variations in ABCC6 associated with PXE are R1141X, exon 23-29 deletion, R1164X, and Q378X, respectively [7]. $\mathrm{R} 1141 \mathrm{X}$ is in exon 24 and represents about $30 \%$ of all PXE mutations. AleI-mediated deletions of exons 23-29 (del 23-29) is the second most common alteration and has been found in at least $20 \%$ of the cases. Other recurrent forms of mutations are: nonsense mutations (a codon is changed to a stop codon) Q378X in exon 9, R518X in exon 12, R1164X in exon 24, and a clustering of nonsense mutations in exons 24 and 28 , which are present in the nucleotide-binding domain that are critical for ABCC6 function. Analysis of missense mutations indicated that frequencies of the mutations residing in the domain-domain interfaces are 3.5- to 4.1-fold higher than those of the entire protein [8]. The inheritance pattern reported in most families has been autosomal recessive, but PXE with autosomal dominant inheritance has also been reported [9]. This condition affects approximately 1 in 160,000 people with a female:male ratio of $2: 1$. The mean onset age of this disease is around 13 years, with a peak number of new cases between 10 and 15 years.

Typically, PXE appears with a formation of yellow papules containing abnormally calcified elastic fibers. Cutaneous lesions often appear in the neck, axilla, cubital fossa, inguinal region, and periumbilical area. Angioid streaks are typically seen in the fundus due to breaks in Bruch's membrane [10]. Finally, clinical delineation of PXE may be confusing because limited manifestations can be detected in some heterozygous carriers, and because cutaneous findings mimicking PXE, or cardiovascular manifestations, can be found in the general population, although at an older age [11]. A recent review questioning autosomal dominant PXE concluded that this mode of inheritance might exist, but it would then be marginal [12].

Similar to CKD, patients affected with PXE have low levels of inorganic pyrophosphate (PPi), a strong inhibitor of ectopic mineralization [13] leading to a typical pattern of progressive calcification of elastic fibers in the skin, eyes, and vasculature [14]. Thus, PXE represents a model disease for arteriosclerosis as it is thought to have an isolated arteriosclerotic type of arterial wall disease with relatively little interference of atherosclerotic arterial wall disease [15].

\section{PXE and Renal Disease}

The renal involvement of PXE has been reported. Several factors are known to promote soft tissue and accelerated arterial calcification in CKD, including systemic inflammation, altered calcium and phosphate homeostasis, and deficiency in endogenous calcification inhibitors. Lau et al. [16] described for the first time that CKD results in acquired $\mathrm{ABCC} 6$ transporter deficiency and may contribute to $\mathrm{CKD}$-associated vascular and soft tissue calcification. On the contrary, although calciphylaxis often occurs in patients with end stage renal disease and secondary hyperparathyroidism, nonuremic calciphylaxis has been described in PXE-affected patients without several forms of CKD [17]. To date, which specific area of the kidney is affected by PXE has been scarcely reported. To our knowledge, in patients with PXE, kidney disease mainly presents as nephrocalcinosis, glomerulonephritis, renovascular hypertension, and nephrolithiasis [18-20]. Histologically, small- to medium-sized renal arteries show elastic fiber fragmentation and mineralization [21]. Interestingly, despite extensive calcification of the renal vasculature reported in some cases, severe forms of CKD have not been described as a consequence of PXE alone.

The bone mineralization process has been investigated in PXE patients. For instance, in a study conducted by Martin et al. [22], patients with PXE were not markedly prone to excessive bone demineralization and risk of 
Fig. 1. Pathophysiological pathways of vascular damage in pseudoxanthoma elasticum (PXE) and chronic kidney disease (CKD). * Alterations found only in CKD patients. $\mathrm{PPi}$, inorganic pyrophosphate. There are several mechanisms involved in the vascular damage in PXE and CKD. From a pathophysiological point of view, the main pathways in PXE are a progressive calcification and fragmentation of elastic fibers with proteoglycan accumulation. Both, PXE and CKD patients express disrupted PPi homeostasis with low levels of this inhibitor of mineralization that leads to a progressive calcification of the vascular bed. Thus, these patients develop increased vascular stiffness in the arterial system. Finally, the bone-vascular axis involvement and factors more closely associated with progressive loss of kidney function (anemia, inflammation, and secondary hyperparathyroidism) contribute to an increased incidence of cardiovascular complications in CKD patients.

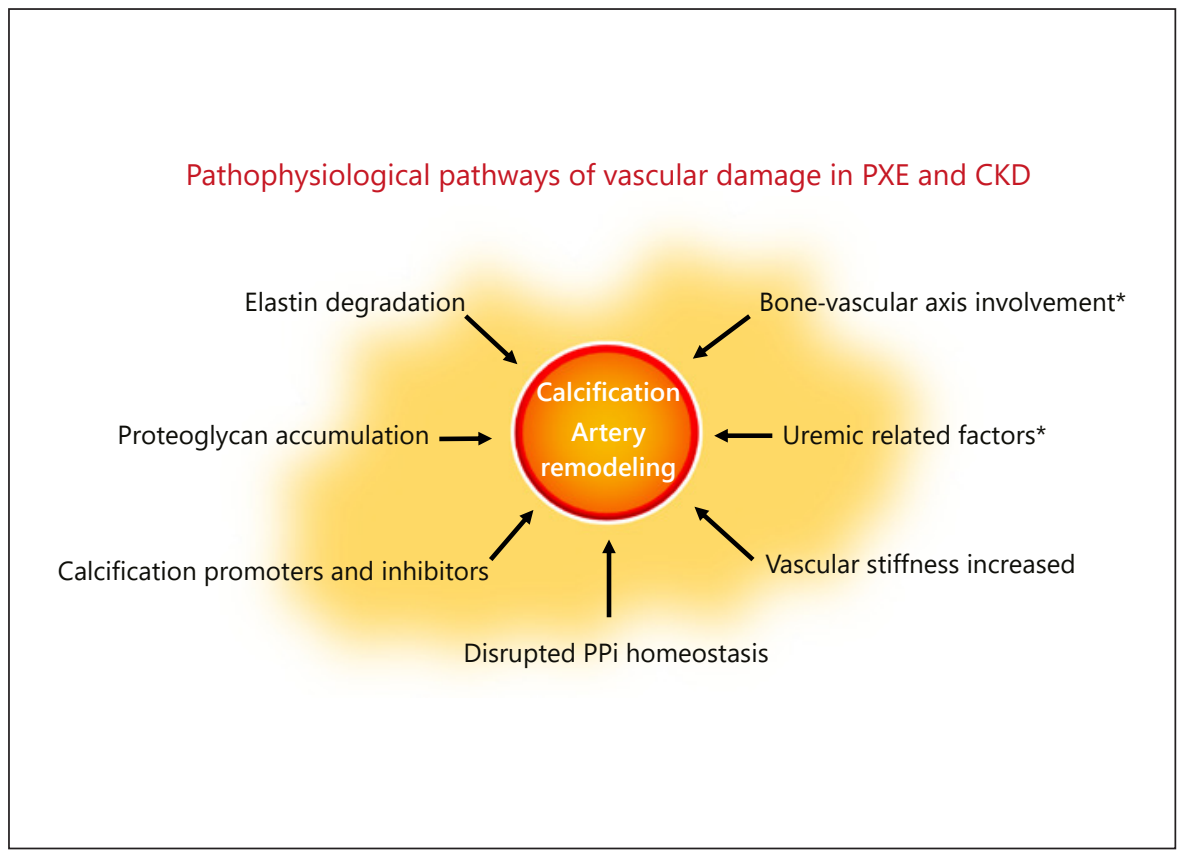

fracture, and, as a consequence, the prevalence of osteoporosis remains as expected for the general population. Furthermore, the relationships between lower-limb arterial calcification, but not coronary artery calcification, and bone mineral density with age are similar to those observed in the general population. Therefore, despite its pivotal role in ectopic calcification, ABCC6 deficiency does not interfere with the bone-vascular axis. The lack of PXE-related disturbances between bone mineral density and arterial calcification also supports vitamin D supplementation in PXE patients with vitamin D deficiency. Of note, patients with moderate or severe CKD always express some degrees of bone demineralization, and the risk of fracture increases dramatically.

\section{Differences in Vascular Patterns}

Although vascular diseases in CKD and PXE share some common patterns, there are specific characteristics to consider (Table 1). An increase in the stiffness of the arterial wall is generally associated with aging and acquired metabolic diseases, such as diabetes and CKD or Monckeberg's atherosclerotic disease [14, 23, 24]. Although the impact of vascular damage on the arterial bed in PXE remains unclear, the impairment in the elastic fiber-rich arterial walls are also involved in the pathology, resulting in precocious and slowly evolving segmental arterial narrowing
Table 1. Differences in the pattern of cardiovascular damage between patients affected with chronic kidney disease (CKD) or pseudoxanthoma elasticum (PXE)

\begin{tabular}{lll}
\hline Factors/disease & CKD & PXE \\
\hline Traditional CVD risk factors & yes & yes \\
Non-traditional CVD risk factors & yes & no \\
Intima layer compromise & yes & no \\
Medial layer compromise & yes & yes \\
Arterial stiffness alteration & yes & yes \\
Mineral bone disease & yes & no \\
\hline
\end{tabular}

CVD, cardiovascular disease.

[25]. Moreover, the internal elastic laminae of small and middle-sized arteries are mainly involved. Thus, the slow course of vessel narrowing is associated with the development of arterial collaterality. Consequently, severe vascular symptoms are infrequent in PXE. Kornet et al. [26] reported that a thicker and more elastic carotid artery was associated with elastin fragmentation and proteoglycan accumulation in PXE patients. Additionally, investigations have shown a tendency to increase the arterial stiffness, and others reduced stiffness or increased wall compressibility [27]. On the contrary, other reports suggested that arterial calcification in PXE is not accompanied by increased stiffness of the arterial wall [28]. 
Table 2. Therapeutic options for vascular damage in patients affected with chronic kidney disease (CKD) or pseudoxanthoma elasticum (PXE)

\begin{tabular}{lll}
\hline Treatment/disease & CKD & PXE \\
\hline Lifestyle modification & yes & yes \\
Pyrophosphate & yes & yes \\
Magnesium & yes & yes \\
TNAP inhibitor & no & yes \\
Phosphate binders/SHPT & yes & yes $/$ no $^{1}$ \\
Vitamin K & yes & yes \\
Hyperaldosteronism & yes & no \\
Bisphosphonates & yes & yes \\
\hline
\end{tabular}

TNAP, tissue-nonspecific alkaline phosphatase; SHPT, secondary hyperparathyroidism.

${ }^{1}$ Limited and conflicting data.

Nevertheless, how relevant could this be in the elderly population or those affected with CKD or diabetes? It is clear that medial-artery calcification contributes to arterial stiffening, which results in hypertension and heart failure, but also pulse pressure-related damage in susceptible highflow end organs like the kidneys [27]. Indeed, increased arterial stiffness is associated with worsening CKD and microvascular damage. To date, the relation of CKD progression in PXE patients has not been well investigated.

As we commented earlier, a complex network of calcification promoters and inhibitors is precisely tuned to inhibit calcification of the internal elastic laminae; thus, PPi is one of the most potent calcification inhibitors [2]. PPi binds to hydroxyapatite crystals inhibiting further growth of the calcifications [29]. As a result, disrupted PPi homeostasis is shown in monogenetic disorders as generalized artery calcification in infancy and arterial calcification due to a deficiency in CD73, PXE, and CKD as well [29]. However, the main difference in the models of vascular damage between PEX and CKD lies in the bone-vascular axis involvement of the latter. Although, the pathophysiological mechanism underlying this phenomenon in CKD is still poorly understood, a large variety of conditions that can modulate the composition of the vascular microenvironment, such as bone turnover, inflammation, or oxidative stress, appears to be mainly responsible (Fig. 1) [2].

In the general population, arterial calcification is heterogeneously distributed across various vascular beds, predominating in the coronary arteries in men and the aorta in women [30]. Compared to other inherited calcifying diseases, the calcification process in PXE is generally considered to be slowly progressive, although exten- sive arterial calcification can develop in infancy [31]. In $\mathrm{CKD}$, this process is accelerated when the glomerular filtration rate is $<60 \mathrm{~mL} / \mathrm{min}$ [32]. The calcification process within the arterial segments of the legs has reported as heterogeneous in PXE, with calcifications predominating in the femoral and subpopliteal arteries [28]. This heterogeneity is probably explained by the underlying pathophysiology, with calcification accumulating preferentially within the middle layers of the below-knee arteries in metabolic diseases and within the intima layer of the proximal arteries, such as the femoral artery, where atheroma predominates [28].

\section{Therapeutic Approaches}

For both, CKD and PXE, the current treatment approach for decelerating CVD is based on the reduction in traditional cardiovascular risk factors through lifestyle changes (smoking cessation, weight loss, daily walking, and moderate physical exercise, etc.) and an appropriate diet with supplemented magnesium, phosphate binders, and pyrophosphate analogues (Table 2) [33]. Moreover, in CKD patients, the nephrologist keeps special attention to uremic-related risk factors like anemia, mineral bone disease, or inflammation, etc.

As in the pathophysiology, CKD and PXE have some therapeutic approaches in common. However, it has not been definitively demonstrated that medical treatment may halt the progression of vascular disease, but several therapies affecting bone and mineral metabolism may help attenuate disease progression [34]. In recent years, therapeutic methodologies to compensate the PPi deficit in animals and humans have been developed successfully [35]. In ABCC6-/- mice, the consumption of PPi-enriched food represents a possible intervention to mitigate calcification progression in PXE. In animal models, new data from Ziegler et al. [36] have shown that ABCC6mutant cells had increased expression and activity of tissue-nonspecific alkaline phosphatase (TNAP), an enzyme that degrades PPi. Hence, a selective and orally bioavailable TNAP inhibitor prevented calcification in these cells in vitro and attenuated both the development and progression of calcification in vivo, without the harmful effects on bone associated with other proposed treatment strategies. This finding offers the rationale that TNAP inhibition would be useful in other disorders of ectopic calcification, including common conditions such as cardiac valve calcification and $\mathrm{VC}$ in $\mathrm{CKD}$, in which decreased PPi has also been documented $[34,37,38]$. 
Improving the abnormalities of mineral metabolism like serum phosphate elevation and parathyroid hormone action has shown significant and independent associations between all-cause mortality and cardiovascular disease end-points in both, patients with normal kidney function and those with CKD. Management of hyperphosphatemia in CKD has involved dietary phosphate restriction, the use of phosphate binders, and dialysis in those with end stage renal disease [39]. Likewise, calcimimetics are used for the treatment of secondary hyperparathyroidism in patients with end stage renal disease. The oral calcimimetic cinacalcet has been associated with reduced VC progression [40]. At this stage, supplementation with $\mathrm{K}$ vitamin as a cofactor of matrix-Gla protein with potential slowing of calcification progression is under investigation [41]. In both human and animal studies, hyperaldosteronism is associated with vascular stiffness, damage, and accelerated atherosclerosis. Thus, research interest has increased in the role of hyperaldosteronism triggering osteoinductive changes in the vasculature [42].

Observational studies suggested that bisphosphonates might provide some protective effects on VC in CKDand PXE-affected patients [43]. Bisphosphonates have anti-inflammatory properties and inhibit the expression of osteogenic proteins that could prove to be beneficial in slowing VC in CKD. The clinical efficiency of etidronate to counteract ectopic mineralization was recently determined in 74 patients with PXE in the TEMP (Treatment of Ectopic Mineralization in Pseudoxanthoma Elasticum) Trial [44]. After a 1-year follow-up, etidronate was found to inhibit arterial media layer calcification and reduce the incidence of ocular complications.

There is accumulating evidence that magnesium is able to suppress phosphate-induced VC in CKD [45]. Magnesium impairs the crystallization of calcium phosphate and, more specifically, the maturation of calciprotein particles. Is well known that phosphate overload causes kidney damage, and magnesium might counteract phosphate toxicity, as in the case of VC. Similarly, the effect of oral magnesium on the progression and severity of PXE has been tested. Although some improvement was noted in patients receiving magnesium supplementation, the results did not reach statistical significance.

Short-chain fatty acid 4-phenylbutyrate (4-PBA), used to treat urea cycle disorders and thalassemia as a nitrogen-scavenging molecule, has been recently employed to promote the maturation of ABCC6 mutants to the plasma membrane. This short-chain fatty acid also influences the transcription of chaperone proteins, and this physiological property has been used in familial intrahepatic cho- lestasis type 2 that results in intracellular retention of an otherwise functional protein. In this regard, in a model of PXE, Pomozi et al. [35] investigated whether 4-PBA administration could block the calcification of ABCC6 mutants. This group employed a cardiac calcification phenotype of Abcc6-/- to quantify the effect of 4-PBA on human ABCC6 mutants expressed in hepatocytes, demonstrating that $4-\mathrm{PBA}$ administration restored the physiological function of ABCC6 mutants, resulting in an enhanced calcification inhibition and identifying the 4-PBA molecule as a promising drug for ABCC6-associated calcification disorders.

Despite intensifying research and increasing awareness of these issues, the underlying pathophysiology of the vasculopathy of CKD and PXE remains mostly unknown. In conclusion, given the impact of these diseases, future investigations may seek additional supportive features to improve and avoid the risk of cardiovascular complications. Moreover, PXE per se represents an attractive model to evaluate the CKD-like vascular damage in non-renal affected models.

\section{Disclosure Statement}

All the authors declare to have no competing interests.

\section{Funding Sources}

There were no funding sources.

\section{Author Contributions}

All authors participated in the design and coordination and helped to draft the manuscript. All authors read and approved the final manuscript.

\section{References}

1 Mizobuchi M, Towler D, Slatopolsky E. Vascular calcification: the killer of patients with chronic kidney disease. J Am Soc Nephrol. 2009 Jul;20(7):1453-64.

2 Evrard S, Delanaye P, Kamel S, Cristol JP, Cavalier E; SFBC/SN joined working group on vascular calcifications. Vascular calcification: from pathophysiology to biomarkers Clin Chim Acta. 2015 Jan;438:401-14.

3 Neldner KH. Pseudoxanthoma elasticum. Clin Dermatol. 1988 Jan-Mar;6(1):1-159.

4 Ringpfeil F, Lebwohl MG, Christiano AM, Uitto J. Pseudoxanthoma elasticum: mutations in the MRP6 gene encoding a transmembrane ATP-binding cassette (ABC) transporter [Internet]. Proc Natl Acad Sci USA. 2000 May;97(11):6001-6.
D’Marco/Lima-Martínez/Karohl/Chacín/ Bermúdez 
5 Viljoen DL, Pope FM, Beighton P. Heterogeneity of pseudoxanthoma elasticum: delineation of a new form? Clin Genet. 1987 Aug; 32(2):100-5.

6 van Soest S, Swart J, Tijmes N, Sandkuijl LA, Rommers J, Bergen AA. A locus for autosomal recessive pseudoxanthoma elasticum, with penetrance of vascular symptoms in carriers, maps to chromosome 16p13.1. Genome Res. 1997 Aug;7(8):830-4.

7 Li Q, Jiang Q, Pfendner E, Váradi A, Uitto J. Pseudoxanthoma elasticum: clinical phenotypes, molecular genetics and putative pathomechanisms. Exp Dermatol. 2009 Jan; 18(1):1-11.

8 Moitra K, Garcia S, Jaldin M, Etoundi C, Cooper D, Roland A, et al. ABCC6 and Pseudoxanthoma Elasticum: The Face of a Rare Disease from Genetics to Advocacy. Int J Mol Sci. 2017 Jul;18(7):E1488.

9 Pope FM. Autosomal dominant pseudoxanthoma elasticum. J Med Genet. 1974 Jun; 11(2):152-7.

$10 \mathrm{Hu}$ X, Plomp AS, van Soest S, Wijnholds J, de Jong PT, Bergen AA. Pseudoxanthoma elasticum: a clinical, histopathological, and molecular update. Surv Ophthalmol. 2003;48(4): 424-38.

11 Chassaing N, Martin L, Calvas P, Le Bert M, Hovnanian A. Pseudoxanthoma elasticum: a clinical, pathophysiological and genetic update including 11 novel ABCC6 mutations. J Med Genet. 2005 Dec;42(12):881-92.

12 Plomp AS, Hu X, de Jong PT, Bergen AA. Does autosomal dominant pseudoxanthoma elasticum exist? Am J Med Genet A. 2004 May;126A(4):403-12.

13 Jansen RS, Duijst S, Mahakena S, Sommer D, Szeri F, Váradi A, et al. ABCC6-mediated ATP secretion by the liver is the main source of the mineralization inhibitor inorganic pyrophosphate in the systemic circulation-brief report. Arterioscler Thromb Vasc Biol. 2014 Sep;34(9):1985-9.

14 Li Q, van de Wetering K, Uitto J. Pseudoxanthoma Elasticum as a Paradigm of Heritable Ectopic Mineralization Disorders: Pathomechanisms and Treatment Development. Am J Pathol. 2019;189(2):216-25.

15 Kranenburg G, Visseren FL, de Borst GJ, de Jong PA, Spiering W; SMART studygroup. Arterial stiffening and thickening in patients with pseudoxanthoma elasticum. Atherosclerosis. 2018;270:160-5.

16 Lau WL, Liu S, Vaziri ND. Chronic kidney disease results in deficiency of ABCC6, the novel inhibitor of vascular calcification. Am J Nephrol. 2014;40(1):51-5.

17 Fernandez KH, Liu V, Swick BL. Nonuremic calciphylaxis associated with histologic changes of pseudoxanthoma elasticum. Am J Dermatopathol. 2013;35(1):106-8.

18 Chraïbi R, Ismaili N, Belgnaoui F, Akallal N, Bouhllab J, Senouci K, et al. Pseudoxanthome élastique et néphrocalcinose. Ann Dermatol Venereol. 2007 Oct;134(10 Pt 1):764-6.
19 Altay M, Turgut F, Karakurt F, Kaya R, Ecemis Z, Gonen N, et al. Membranoproliferative glomerulonephritis associated with pseudoxanthoma elasticum. Int Urol Nephrol. 2008; 40(4):1099-101.

20 Fabre B, Bayle P, Bazex J, Durand D, Lamant L, Chassaing N. Pseudoxanthoma elasticum and nephrolithiasis. J Eur Acad Dermatol Venereol. 2005 Mar; 19(2):212-5.

21 Vasudevan B, Shijith KP, Bahal A, Raghav V. Pseudoxanthoma Elasticum with Renal Cortical Calcification. Med J Armed Forces India. $2010 \mathrm{Jul} ; 66(3): 272-4$.

22 Martin L, Hoppé E, Kauffenstein G, Omarjee L, Navasiolava N, Henni S, et al. Early arterial calcification does not correlate with bone loss in pseudoxanthoma elasticum. Bone. 2017 Oct; $103: 88-92$.

23 Amann K. Media calcification and intima calcification are distinct entities in chronic kidney disease. Clin J Am Soc Nephrol. 2008 Nov;3(6):1599-605.

24 David Smith C, Gavin Bilmen J, Iqbal S, Robey S, Pereira M. Medial artery calcification as an indicator of diabetic peripheral vascular disease. Foot Ankle Int. 2008 Feb;29(2): $185-90$.

25 Mendelsohn G, Bulkley BH, Hutchins GM. Cardiovascular manifestations of Pseudoxanthoma elasticum. Arch Pathol Lab Med. 1978 Jun;102(6):298-302.

26 Kornet L, Bergen AA, Hoeks AP, Cleutjens JP, Oostra RJ, Daemen MJ, et al. In patients with pseudoxanthoma elasticum a thicker and more elastic carotid artery is associated with elastin fragmentation and proteoglycans accumulation. Ultrasound Med Biol. 2004 Aug; 30(8):1041-8

27 Bartstra JW, de Jong PA, Spiering W. Accelerated peripheral vascular aging in pseudoxanthoma elasticum - proof of concept for arterial calcification-induced cardiovascular disease. Aging (Albany NY). 2019;11(3):1062-4.

28 Leftheriotis G, Kauffenstein G, Hamel JF, Abraham P, Le Saux O, Willoteaux S, et al. The contribution of arterial calcification to peripheral arterial disease in pseudoxanthoma elasticum. PLoS One. 2014;9(5):e96003.

29 Lanzer P, Boehm M, Sorribas V, Thiriet M, Janzen J, Zeller T, et al. Medial vascular calcification revisited: review and perspectives. Eur Heart J. 2014 Jun;35(23):1515-25.

30 Nasir K, Roguin A, Sarwar A, Rumberger JA, Blumenthal RS. Gender differences in coronary arteries and thoracic aorta calcification. Arterioscler Thromb Vasc Biol. 2007 May; 27(5):1220-2.

31 Le Boulanger G, Labrèze C, Croué A, Schurgers LJ, Chassaing N, Wittkampf T, et al. An unusual severe vascular case of pseudoxanthoma elasticum presenting as generalized arterial calcification of infancy. Am J Med Genet A. 2010 Jan;152A(1):118-23.

32 Drüeke TB, Massy ZA. Atherosclerosis in CKD: differences from the general population. Nat Rev Nephrol. 2010;6(12):723-35.
33 Germain DP. Pseudoxanthoma elasticum. Orphanet J Rare Dis. 2017 May;12(1):85.

34 Ureña-Torres P, D’Marco L, Raggi P, GarcíaMoll X, Brandenburg V, Mazzaferro S, et al. Valvular heart disease and calcification in CKD: more common than appreciated. Nephrol Dial Transplant. 2019 Jul; Available from: http://www.ncbi.nlm.nih.gov/ pubmed/31326992.

35 Pomozi V, Julian CB, Zoll J, Pham K, Kuo S, Tőkési N, et al. Dietary Pyrophosphate Modulates Calcification in a Mouse Model of Pseudoxanthoma Elasticum: Implication for Treatment of Patients. J Invest Dermatol. 2019 May;139(5):1082-8.

36 Ziegler SG, Ferreira CR, MacFarlane EG, Riddle RC, Tomlinson RE, Chew EY, et al. Ectopic calcification in pseudoxanthoma elasticum responds to inhibition of tissue-nonspecific alkaline phosphatase. Sci Transl Med. 2017 Jun;9(393):eaal1669.

37 O'Neill WC, Sigrist MK, McIntyre CW. Plasma pyrophosphate and vascular calcification in chronic kidney disease. Nephrol Dial Transplant. 2010 Jan;25(1):187-91.

38 Rathan S, Yoganathan AP, O’Neill CW. The role of inorganic pyrophosphate in aortic valve calcification. J Heart Valve Dis. 2014 Jul; 23(4):387-94.

39 Block GA. Therapeutic interventions for chronic kidney disease-mineral and bone disorders : focus on mortality. Curr Opin Nephrol Hypertens. 2011 Jul;20(4):376-381.

40 Raggi P, Chertow GM, Torres PU, Csiky B, Naso A, Nossuli K, et al. The ADVANCE study: A randomized study to evaluate the effects of cinacalcet plus low-dose vitamin $\mathrm{D}$ on vascular calcification in patients on hemodialysis. Nephrol Dial Transplant. 2011;26(4): 1327-39.

41 Rattazzi M, Bertacco E, Del Vecchio A, Puato M, Faggin E, Pauletto P. Aortic valve calcification in chronic kidney disease. Nephrol Dial Transplant. 2013;28(12):2968-76.

42 Ruderman I, Holt SG, Hewitson TD, Smith ER, Toussaint ND. Current and potential therapeutic strategies for the management of vascular calcification in patients with chronic kidney disease including those on dialysis. Semin Dial. 2018;31(5):487-99.

43 Sterbakova G, Vyskocil V, Linhartova K. Bisphosphonates in calcific aortic stenosis: association with slower progression in mild disease-a pilot retrospective study. Cardiology. 2010;117(3):184-9.

44 Kranenburg G, de Jong PA, Bartstra JW, Lagerweij SJ, Lam MG, Ossewaarde-van Norel J, et al. Etidronate for Prevention of Ectopic Mineralization in Patients with Pseudoxanthoma Elasticum. J Am Coll Cardiol. 2018 Mar;71(10):1117-26.

45 Sakaguchi Y, Hamano T, Isaka Y. Magnesium and Progression of Chronic Kidney Disease: Benefits Beyond Cardiovascular Protection? Adv Chronic Kidney Dis. 2018 May;25(3): $274-80$ 